\title{
29. CHEMICAL ZONATION OF PLAGIOCLASE PHENOCRYSTS FROM LEG 51, 52, AND 53 BASALTS
}

Claire Bollinger and Michel Semet, Laboratoire de Géochimie et Cosmochimie, Institut de Physique du Globe de Paris, Département des Sciences de la Terre, 75230 Paris, France

\section{INTRODUCTION}

Chemical zonation of phenocryst phases present a record of the physicochemical changes in magma from which they crystallized. Several recent studies (e.g., Bottinga et al., 1966; Albarède and Bottinga, 1972; Kirkpatrick, 1976, 1977; Sibley et al., 1976; Shimizu, 1978) have addressed the zonation of major and trace elements in plagioclase from the point of view of the growth dynamics from a magma and have shown that disequilibrium composition features of the phenocrysts should provide a better understanding of the physicochemical evolution of the associated magma. As diffusion coefficients, growth rates, and chemical composition of phenocrysts as a function of supercooling become available, parameters like cooling rates and non-equilibrium partition of trace elements of crystallizing magmas may be modeled quantitatively. Oceanic basalts are among the terrestrial volcanic rocks that show relatively little differentiation from primitive liquids originating in the mantle and do not seem to show extensive mixing from different sources. Their relatively simple crystallization history make them well suited for the detailed study of the chemical zonation of their phenocryst assemblages.

The types of zonation patterns found in plagioclase phenocrysts from volcanic rocks and their possible petrogenetic interpretation are reviewed by Smith (1974, chapt. 14 to 17). Four main types are present in plagioclase phyric oceanic basalts: continuous zoning, oscillatory zoning, patchy zoning, and sector zoning. Some phenocrysts may show more than one type in the different growth zones, indicating that several growth-controlled phenomena may be at work during the crystallization of a single crystal. Furthermore, correlation of zonation patterns between the growth faces of individual phenocrysts, or between different crystals of the same sample (e.g., Sibley et al., 1976), may help in attributing the patterns to specific growth features such as chemical changes in the liquids, cooling rate changes, settling, floating, and convection in the magma.

Samples were selected from Legs 51,52 , and 53 of Glomar Challenger for which a thick section of wellcharacterized basalts was available. The aim of our study is to obtain detailed major and trace element data for several selected samples of the basalt pile, with the hope of reconstructing some aspects of their petrogenetic history. This report is concerned primarily with zonation patterns of the major elements ( $\mathrm{Na}, \mathrm{Al}, \mathrm{Si}$, and $\mathrm{Ca}$ ) and is preliminary in several regards; major element data can provide only limited information on the zonation patterns and, more importantly, the resolution in the chemical changes is limited to several $\mu \mathrm{m}$ by electron microprobe analysis. The second part of the study consists of detailed trace element determinations in the samples studied by ion microprobe analysis. A comprehensive discussion of the results, therefore, will not be attempted at this point.

\section{ANALYTICAL PROCEDURE}

Most samples studied are mino-cores. Doubly polished thin sections were prepared by standard petrographic techniques. Chemical analyses were performed with a CAMECA Camebax electron microprobe which has a $40^{\circ}$ take-off angle. Wavelength dispersive spectrometers and a primary current of 0.01 to $0.04 \mu \mathrm{A}$ were routinely used. The beam was slightly defocused (3-5 $\mu \mathrm{m} \emptyset)$ for large zones, whereas spot analysis $(\leqslant 1 \mu \mathrm{m}$ spot $\varnothing)$ was carried out for the finer zones. A modified Bence-Albee data reduction program (Bence and Albee, 1968) utilizing a Lake County labradorite, an Amelia County albite, and an Eifel (Germany) orthoclase standard was used to obtain the major oxide concentrations.

\section{RESULTS}

Most of the samples studied were from pillow basalts, although a few came from massive units. Preliminary petrographic examination showed that zonation patterns were of three general types: (a) relatively homogeneous crystals; (b) oscillatory zoned crystals; (c) oscillatory and sector zoned crystals.

Phenocrysts from pillow basalts may be of any of the three types; type b is, however, the most abundant. Massive units, on the contrary, mostly contain type b phenocrysts. All phenocrysts have a relatively sodic rim of variable thickness ranging from 10 to $100 \mu \mathrm{m}$.

No systematic differences between the samples coming from different depths in the boreholes were found. The most representative samples were, therefore, selected for the microprobe study (see Table 1). The phenocrysts are either subequant or lath-shaped. Prominent growth was along [001], [110], and [201] faces. Patchy zoning is apparently restricted to the cores of the phenocrysts. Bands rich in glassy inclusions may occur anywhere in the crystal and are continuous around it. Oscillatory zones generally show two thickness ranges. Larger zones, tens of $\mu \mathrm{m}$ wide, are subdivided into $\mu \mathrm{m}$ size zones. The latter cannot be compositionally resolved with the electron microprobe. Correlation of the larger zones between different growing faces, or symmetry related faces, is perfect in most cases. A major proportion of the oscillatory zones are planar and parallel to growing faces. However, a few samples show wavy or contorted bands not conforming to prominent faces. Engulfment of inclusions does not seem to be the 
TABLE 1

Petrographic Descriptions

\begin{tabular}{|c|c|c|c|c|}
\hline Leg & Hole & Sample & Unit & Description \\
\hline 51 & $417 \mathrm{D}$ & $\begin{array}{l}33-4 \\
12 \mathrm{~cm}\end{array}$ & - & $\begin{array}{l}\text { Massive basalt, groundmass is coarse } \\
\text { grained, containing oxides; zoned } \\
\text { plagioclase phenocrysts up to } 4 \mathrm{~mm} \text {; } \\
\text { crystal types b and c }\end{array}$ \\
\hline 52 & $418 \mathrm{~A}$ & $\begin{array}{l}28-2 \\
10 \mathrm{~cm}\end{array}$ & 5 & $\begin{array}{l}\text { Moderately porphyritic pillow basalt } \\
\text { with olivine, pyroxene, and plagio- } \\
\text { clase phenocyrsts; groundmass is } \\
\text { slightly altered; a spinel inclusion } \\
\text { in plagioclase phenocryst; crystal } \\
\text { types b and c }\end{array}$ \\
\hline 53 & $418 \mathrm{~A}$ & $\begin{array}{c}50-2, \\
105 \mathrm{~cm}\end{array}$ & $6 \mathrm{~b}$ & $\begin{array}{l}\text { Moderately porphyritic pillow basalt } \\
\text { with olivine and plagioclase pheno- } \\
\text { crysts, fine-grained groundmass; } \\
\text { plagioclase is fresh and contains al- } \\
\text { tered glass inclusions; crystal types } \\
\text { a, b, c }\end{array}$ \\
\hline 53 & $418 \mathrm{~A}$ & $\begin{array}{l}71-2, \\
52 \mathrm{~cm}\end{array}$ & $11 \mathrm{f}$ & $\begin{array}{l}\text { Pillow basalt moderately porphyritic } \\
\text { with olivine and plagioclase pheno- } \\
\text { crysts and aphyric matrix; crystal } \\
\text { types b and c }\end{array}$ \\
\hline 53 & $418 \mathrm{~A}$ & $\begin{array}{l}71-2 \\
66 \mathrm{~cm}\end{array}$ & $11 f$ & $\begin{array}{l}\text { Glassy zones from a pillow basalt } \\
\text { with } 3 \mathrm{~mm} \text { plagioclase phenocrysts; } \\
\text { glass inclusions in the phenocrysts are } \\
\text { mostly fresh; cyrstal types b and c }\end{array}$ \\
\hline 53 & $418 \mathrm{~A}$ & $\begin{array}{l}80-2, \\
55 \mathrm{~cm}\end{array}$ & 12 & $\begin{array}{l}\text { Massive basalt with plagioclase and } \\
\text { olivine phenocrysts and very coarse } \\
\text { grained matrix; crystal types b and c }\end{array}$ \\
\hline 53 & $418 \mathrm{~A}$ & $\begin{array}{l}85-2, \\
93 \mathrm{~cm}\end{array}$ & 12 & $\begin{array}{l}\text { Massive basalt with olivine, plagio- } \\
\text { clase, and pyroxene phenocrysts and } \\
\text { coarse-grained matrix; crystal type b }\end{array}$ \\
\hline 53 & $418 \mathrm{~A}$ & $\begin{array}{l}86-3 \\
93 \mathrm{~cm}\end{array}$ & 13 & $\begin{array}{l}\text { Pillow basalt with olivine, plagioclase, } \\
\text { and pyroxene phenocrysts and very } \\
\text { fine-grained matrix; crystal types b } \\
\text { and c }\end{array}$ \\
\hline
\end{tabular}

general explanation for this feature for which no rationale has been found.

Analytical data for the major elements are presented in Table 2. A summary of the results is illustrated in Figure 1 which presents a plot of $\mathrm{Ca}$ versus $\mathrm{Al}$ atom number for analyses normalized to 8 oxygens; this type of illustration follows Bryan (1974). Detailed analyses for $\mathrm{K}, \mathrm{Mg}$, and $\mathrm{Fe}$ are not presented at this point because these elements will be determined with higher accuracy with an ion microprobe. Preliminary ion microprobe results show that $\mathrm{K}$ is generally on the order of $100 \mathrm{ppm}$ weight and that $\mathrm{Mg}$ and $\mathrm{Fe}$ increase from core to rim and are enriched in $\{010\}$ sectors as observed by Bryan (1974). The average composition of the plagioclase is An75-80 and is relatively more calcic than those described by Bryan (1974). For those analyses where K, $\mathrm{Mg}$, and $\mathrm{Fe}$ are reported, the total number of cations is close to the theoretical 5 to 8 oxygens so that extensive nonstoichiometry does not seem to be present (Figure 2).

\section{DISCUSSION}

The $\mathrm{Ca}$ versus $\mathrm{Al}$ relationship of Figure 1 generally follows the one reported by Bryan (1974) in showing enrichment of $\mathrm{Ca}$ relative to the theoretical Ab-An solidsolution series. Our data lie closer to the theoretical line; solid solution of the hypothetical end-member $\mathrm{Ca}(\mathrm{Mg}, \mathrm{Fe})$ $\mathrm{Si}_{3} \mathrm{O}_{8}$, as suggested by the latter author accounts satisfactorily for the shift. Linear fits to the data for pillow basalts and massive flows are slightly different, but within the limits of error of the least-squares fitted best lines.

Thermodynamics indicate that the most calcic compositions are likely to be closer to equilibrium composition for the crystallization of the plagioclases. Had equilibrium been maintained, plagioclases of An85-90 composition should have crystallized from the basaltic melt. Departure from this composition in the larger oscillatory zoned bands are taken to reflect different degrees of undercooling. A crude estimate of this parameter may be obtained by comparison of the plagioclase composition to that observed under controlled temperature conditions for magmas of similar bulk composition. Undercoolings of $50^{\circ}$ to $100^{\circ} \mathrm{C}$ gave rise to An65 plagioclases in the Makaopuhi lava lake (Wright and Kinoshita, 1968), whereas Clochiatti et al. (1978) obtained $\mathrm{An}_{65}$ and $\mathrm{An}_{51}$ plagioclases for $30^{\circ}$ and $100^{\circ} \mathrm{C}$ undercoolings, respectively. Those observations then suggest that the degree of undercooling prevailing during crystallization of the phenocrysts in the oceanic basalts was less than a few tens of degrees and possibly as low as a few degrees C. Crystallization of the sodic rims may have occurred at greater undercoolings of $50^{\circ}$ to $100^{\circ} \mathrm{C}$, and have corresponded to eruption on the ocean floor. Enhanced nucleation rates at this stage are in keeping with the appearance of microphenocrysts of similar composition. Even though other phenomena may play a role in the development of the larger oscillatory zoned bands, variations in cooling rate of the body of magma that crystallized the phenocrysts in a step-by-step progression into colder rocks seem to afford an adequate explanation. The variations would induce variable degrees of constitutional supercooling in the sense of Bottinga et al. (1966) and Sibley et al. (1976). In this line of reasoning, the fine $\mu \mathrm{m}$-size zones remain inadequately explained. They may be due to the build-up of "poisons" at the growing surface owing to their slow diffusion in the liquid.

A fair conclusion from the data at hand would be that disequilibrium growth effects, such as those suggested by Albarède and Bottinga (1972), are not pronounced. However, our contention is that the scale of the latter phenomenon is generally below the resolution of microprobe analysis. The mere fact that sectors have different average compositions and that growth zones for different crystallographic faces do not always correspond strongly suggest that growth rate is an important parameter in controlling the composition of the growing layer.

\section{ACKNOWLEDGMENTS}

The study has been supported in part by a grant from the CNRS (ATP "Gèochimie"). C.B. benefited from a fellowship of the Centre National pour l'Exploitation des Océans. The manuscript was improved by the thoughtful comments of Dr. S. Wilhelm and of anonymous reviewers.

\section{REFERENCES}

Albarède, F. and Bottinga, Y., 1972. Kinetic disequilibrium in trace element partitioning between phenocrysts and host lava, Geochim. Cosmochim. Acta., v. 136, p. 141-156.

Bence, A.E. and Albee, A.L., 1968. Empirical correction factors for the electron microanalysis of silicates and oxides, J. Geol., v. 76, p. 382 . 
TABLE 2

Analytical Data for Major Elements

\begin{tabular}{|c|c|c|c|c|c|c|c|c|c|c|c|c|c|c|c|c|c|c|c|c|c|c|c|}
\hline & \multicolumn{23}{|c|}{ Sample 51-417D-33-4 } \\
\hline & 1 & 2 & 3 & 4 & 5 & 6 & 7 & 8 & 9 & 10 & 11 & 12 & 13 & 14 & 15 & 16 & 17 & 18 & 19 & 20 & 21 & 22 & 23 \\
\hline $\mathrm{SiO}_{2}$ & 51.79 & 48.44 & 47.99 & 48.19 & 49.03 & 48.85 & 50.57 & 46.60 & 50.69 & 46.32 & 46.68 & 48.15 & 47.74 & 47.98 & 48.15 & 51.76 & 56.41 & 51.24 & 47.50 & 54.73 & 46.62 & 47.02 & 47.11 \\
\hline $\mathrm{Al}_{2} \mathrm{O}_{3}$ & 30.02 & 33.40 & 33.51 & 33.47 & 32.68 & 32.83 & 31.51 & 34.69 & 31.23 & 34.64 & 34.54 & 33.60 & 34.03 & 33.70 & 33.53 & 30.95 & 27.28 & 30.33 & 34.21 & 28.35 & 34.51 & 34.18 & 33.79 \\
\hline $\mathrm{CaO}^{2}-\mathrm{O}^{2}$ & 13.64 & 16.61 & 16.49 & 16.73 & 16.52 & 16.18 & 14.89 & 18.44 & 14.49 & 17.80 & 17.62 & 16.91 & 17.13 & 16.94 & 16.77 & 14.46 & 10.68 & 14.26 & 17.19 & 11.47 & 18.03 & 17.75 & 17.22 \\
\hline $\mathrm{Na}_{2} \mathrm{O}$ & 3.68 & 1.89 & 1.86 & 1.84 & 2.10 & 2.40 & 2.84 & 1.19 & 3.27 & 1.17 & 1.32 & 2.03 & 1.63 & 2.04 & 2.09 & 3.38 & 5.65 & 3.11 & 1.48 & 5.15 & 1.27 & 1.51 & 1.48 \\
\hline $\mathrm{K}_{2} \mathrm{O}$ & 0.09 & 0.04 & 0.03 & 0.03 & 0.06 & 0.04 & 0.05 & 0.03 & 0.07 & 0.06 & 0.03 & 0.04 & 0.04 & 0.02 & 0.04 & 0.04 & 0.06 & 0.08 & 0.04 & 0.06 & 0.04 & 0.03 & 0.05 \\
\hline $\mathrm{FeO}$ & 0.85 & 0.41 & 0.41 & 0.35 & 0.34 & 0.37 & 0.52 & 0.33 & 0.63 & 0.38 & 0.45 & 0.42 & 0.41 & 0.41 & 0.34 & 0.58 & 0.98 & 0.75 & 0.55 & 0.93 & 0.38 & 0.47 & 0.78 \\
\hline Total & 100.07 & 100.69 & 100.29 & 100.61 & 100.73 & 100.67 & 100.38 & 101.28 & 100.38 & 100.37 & 100.64 & 101.15 & 100.98 & 101.19 & 100.92 & 101.17 & 101.06 & 99.77 & 100.97 & 100.85 & 100.96 & 100.85 & 100.43 \\
\hline \multicolumn{24}{|c|}{$\begin{array}{l}\text { Nb cations } \\
\text { per } 8 \text { oxygens }\end{array}$} \\
\hline Si & 2.359 & 2.204 & 2.193 & 2.196 & 2.230 & 2.224 & 2.298 & 2.121 & 2.306 & 2.124 & 2.134 & 2.187 & 2.171 & 2.181 & 2.190 & 2.333 & 2.524 & 2.341 & 2.161 & 2.464 & 2.129 & 2,145 & 2.159 \\
\hline Al & 1.612 & 1.791 & 1.805 & 1.797 & 1.752 & 1.761 & 1.688 & 1.861 & 1.674 & 1.872 & 1.861 & 1.798 & 1.824 & 1.805 & 1.797 & 1.644 & 1.438 & 1.633 & 1.835 & 1.504 & 1.858 & 1.838 & 1.825 \\
\hline $\mathrm{Ca}$ & 0.666 & 0.805 & 0.807 & 0.817 & 0.805 & 0.789 & 0.725 & 0.899 & 0.706 & 0.875 & 0.863 & 0.823 & 0.835 & 0.725 & 0.817 & 0.698 & 0.512 & 0.698 & 0.838 & 0.553 & 0.882 & 0.868 & 0.846 \\
\hline $\mathrm{Na}$ & 0.325 & 0.167 & 0.165 & 0.163 & 0.185 & 0.212 & 0.200 & 0.105 & 0.288 & 0.104 & 0.117 & 0.179 & 0.144 & 0.180 & 0.184 & 0.295 & 0.490 & 0.276 & 0.131 & 0.450 & 0.112 & 0.134 & 0.132 \\
\hline $\begin{array}{l}\mathrm{K} \\
\mathrm{Mg}\end{array}$ & 0.005 & 0.002 & 0.002 & 0.002 & 0.003 & 0.002 & 0.003 & 0.002 & 0.004 & 0.004 & 0.002 & 0.002 & 0.002 & 0.001 & 0.002 & 0.002 & 0.003 & 0.005 & 0.002 & 0.003 & 0.002 & 0.002 & 0.003 \\
\hline $\begin{array}{l}\mathrm{Mg} \\
\mathrm{Fe}\end{array}$ & 0.032 & 0.016 & 0.016 & 0.013 & 0.013 & 0.014 & 0.020 & 0.013 & 0.024 & 0.015 & 0.017 & 0.016 & 0.016 & 0.016 & 0.013 & 0.022 & 0.037 & 0.029 & 0.021 & 0.035 & 0.015 & 0.018 & 0.020 \\
\hline Total & 5.000 & 4.985 & 4.988 & 4.988 & 4.988 & 5.003 & 4.984 & 5.001 & 5.003 & 4.993 & 4.995 & 5.005 & 4.990 & 5.007 & 5.004 & 4.994 & 5.004 & 4.982 & 4.988 & 5.010 & 4.999 & 5.004 & 4.995 \\
\hline \multirow[t]{3}{*}{$\%$ An } & 64.72 & 81.31 & 81.52 & 82.11 & 80.02 & 77.58 & 72.65 & 88.22 & 69.08 & 87.67 & 86.39 & 80.69 & 83.75 & 80.72 & 80.41 & 68.63 & 49.13 & 69.25 & 84.48 & 53.12 & 87.24 & 84.93 & 83.68 \\
\hline & \multicolumn{23}{|c|}{ Sample 53-418A-71-2 } \\
\hline & 24 & 25 & 26 & 27 & 28 & 29 & 30 & 31 & 32 & 33 & 34 & 35 & 36 & 37 & 38 & 39 & 40 & 41 & 42 & 43 & 44 & 45 & 46 \\
\hline $\mathrm{SiO}_{2}$ & 54.61 & 49.71 & 50.68 & 48.31 & 52.54 & 49.43 & 50.26 & 47.31 & 47.95 & 53.29 & 52.20 & 51.42 & 49.43 & 51.55 & 50.40 & 49.47 & 47,06 & 47.16 & 53.32 & 52.03 & 52.39 & 48.00 & 48.83 \\
\hline $\mathrm{Al}_{2} \mathrm{O}_{3}$ & 27.36 & 32.21 & 31.82 & 33.35 & 29.91 & 32.12 & 31.94 & 33.58 & 32.80 & 28.61 & 30.36 & 30.36 & 31.77 & 30.84 & 31.23 & 32.06 & 34.01 & 33.62 & 28.25 & 30.28 & 29.74 & 32.88 & 31.50 \\
\hline $\mathrm{CaO}$ & 10.55 & 15.51 & 15.50 & 16.52 & 14.24 & 15.95 & 15.26 & 18.00 & 17.54 & 13.52 & 13.96 & 14.09 & 15.82 & 14.44 & 14.12 & 14.98 & 17.66 & 17.21 & 13.06 & 14.58 & 14.11 & 16.94 & 16.35 \\
\hline $\mathrm{Na}_{2} \mathrm{O}$ & 5.12 & 2.48 & 2.64 & 2.09 & 3.31 & 2.24 & 2.45 & 1.39 & 1.76 & 3.77 & 3.19 & 3.13 & 2.37 & 2.95 & 2.72 & 2.37 & 1.24 & 1.30 & 3.92 & 3.28 & 3.37 & 1.65 & 2.02 \\
\hline $\begin{array}{l}\mathrm{K}_{2} \mathrm{O} \\
\mathrm{MgOO}\end{array}$ & 0.08 & 0.05 & 0.06 & 0.01 & 0.04 & 0.02 & 0.05 & 0.03 & 0.03 & 0.06 & 0.05 & 0.05 & 0.03 & 0.01 & 0.05 & 0.03 & 0.04 & 0.04 & 0.08 & 0.05 & 0.05 & 0.01 & 0.05 \\
\hline $\begin{array}{l}\mathrm{MgO} \\
\mathrm{FeO}\end{array}$ & 1.07 & 0.50 & 0.49 & 0.33 & 0.76 & 0.48 & 0.46 & 0.41 & 0.41 & 0.78 & 0.59 & 0.63 & 0.46 & 0.59 & 0.41 & 0.31 & 0.34 & 0.28 & 0.96 & 0.59 & 0.54 & 0.45 & 0.54 \\
\hline Total & 98.79 & 100.46 & 101.19 & 100.61 & 100.80 & 100.24 & 100.42 & 100.72 & 100.49 & 100.03 & 100.36 & 99.68 & 99.88 & 100.38 & 98.93 & 99.22 & 100.35 & 99.61 & 99.59 & 100.81 & 100.20 & 99.93 & 99.29 \\
\hline \multicolumn{24}{|c|}{$\begin{array}{l}\text { Nb cations } \\
\text { per } 8 \text { oxygens }\end{array}$} \\
\hline $\mathrm{Si}$ & 2.499 & 2.262 & 2.288 & 2.201 & 2.371 & 2.256 & 2.283 & 2.163 & 2.195 & 2.422 & 2.36 & 2.348 & 2.264 & 2.3 & 2.315 & 2.271 & 2.155 & 2.172 & 2.434 & 2.353 & 2.378 & 2.204 & 2.254 \\
\hline Al & 1.476 & 1.727 & 1.693 & 1.791 & 1.593 & 1.727 & 1.710 & 1.809 & 1.770 & 1.533 & 1.620 & 1.634 & 1.715 & 1.648 & 1.691 & 1.735 & 1.836 & 1.825 & 1.520 & 1.614 & 1.591 & 1.779 & 1.714 \\
\hline $\mathrm{Ca}$ & 0.517 & 0.756 & 0.750 & 0.806 & 0.689 & 0.780 & 0.743 & 0.882 & 0.860 & 0.658 & 0.678 & 0.689 & 0.776 & 0.701 & 0.695 & 0.737 & 0.867 & 0.849 & 0.639 & 0.706 & 0.686 & 0.833 & 0.809 \\
\hline $\mathrm{Na}$ & 0.454 & 0.219 & 0.231 & 0.185 & 0.290 & 0.198 & 0.216 & 0.123 & 0.156 & 0.333 & 0.280 & 0.277 & 0.210 & 0.260 & 0.242 & 0.211 & 0.110 & 0.116 & 0.347 & 0.288 & 0.297 & 0.147 & 0.181 \\
\hline $\mathrm{K}$ & 0.005 & 0.003 & 0.003 & 0.001 & 0.002 & 0.001 & 0.003 & 0.002 & 0.002 & 0.003 & 0.003 & 0.003 & 0.002 & 0.001 & 0.003 & 0.002 & 0.002 & 0.002 & 0.005 & 0.003 & 0.003 & 0.001 & 0.003 \\
\hline $\begin{array}{l}\mathrm{Mg}_{\mathrm{g}} \\
\mathrm{Fe}_{e}\end{array}$ & 0.041 & 0.019 & 0.018 & 0.013 & 0.025 & 0.018 & 0.017 & 0.016 & 0.016 & 0.030 & 0.022 & 0.024 & 0.018 & 0.022 & 0.016 & 0.012 & 0.013 & 0.011 & 0.037 & 0.022 & 0.021 & 0.017 & 0.021 \\
\hline Total & 4.922 & 4.986 & 4.983 & 4.996 & 4.976 & 4.980 & 4.972 & 4.995 & 4.999 & 4.979 & 4.967 & 4.975 & 4.985 & 4.969 & 4.962 & 4.968 & 4.983 & 4.975 & 4.982 & 4.986 & 4.976 & 4.981 & 4.981 \\
\hline$\%$ An & 50.84 & 75.83 & 74.85 & 80.36 & 68.22 & 78.24 & 75.89 & 86.3 & 83.17 & 64.32 & 68.97 & 69.39 & 77.14 & 71.24 & 72.70 & 76.61 & 87.40 & 86.81 & 62.16 & 69.28 & 68.12 & 83.47 & 79.86 \\
\hline
\end{tabular}




\begin{tabular}{|c|c|c|c|c|c|c|c|c|c|c|c|c|c|c|c|c|c|c|c|c|c|c|c|}
\hline & \multicolumn{7}{|c|}{ Sample $52-418 A-71-2$} & \multicolumn{2}{|c|}{$\begin{array}{l}\text { Sample } 52-418 \mathrm{~A}- \\
71-2,60-69 \mathrm{~cm}\end{array}$} & \multicolumn{2}{|c|}{$\begin{array}{l}\text { Sample } 52-418 \mathrm{~A}- \\
80-2,55-58 \mathrm{~cm}\end{array}$} & \multicolumn{12}{|c|}{ Sarmple $53-417 \mathrm{~A}-86-3$} \\
\hline & 47 & 48 & 49 & so & 51 & 52 & 53 & 54 & 55 & 56 & 57 & 58 & 59 & 60 & 61 & 62 & 63 & 64 & 65 & 66 & 67 & 68 & 69 \\
\hline $\mathrm{SiO}_{2}$ & 48.22 & 50.37 & 49.36 & 52.23 & 48.38 & 48.63 & 48.54 & 50.55 & 47.66 & 49.68 & 46.12 & 53.59 & 53.30 & 50.25 & 47.17 & 48.42 & 48.98 & 53.83 & 51.97 & 55.13 & 50.99 & 47.36 & 47.58 \\
\hline $\mathrm{Al}_{2} \mathrm{O}_{3}$ & 31.92 & 31.12 & 32.09 & 29.21 & 31.83 & 31.94 & 32.42 & 30.45 & 31.99 & 31.52 & 33.21 & 28.74 & 29.11 & 30.91 & 34.11 & 32.63 & 32.58 & 28.53 & 30.72 & 27.68 & 30.44 & 3.03 & 32.48 \\
\hline $\mathrm{CrO}^{\circ}$ & 17.13 & 15.84 & 16.57 & 13.28 & 15.73 & 16.58 & 16.78 & 14.29 & 15.90 & 15.43 & 17.50 & 11.89 & 12.86 & 14.86 & 17,90 & 16.33 & 16.52 & 11.83 & 14.13 & 11.41 & 15.05 & 17.04 & 17.03 \\
\hline $\mathrm{Na}_{2} \mathrm{O}$ & 1.88 & 2.71 & 2.17 & 3.41 & 2.19 & 2.06 & 1.84 & 2.98 & 2.06 & 2.67 & 1.81 & 4.73 & 4.32 & 2.89 & 1.53 & 2.35 & 2.33 & 5.06 & 3.49 & 5.31 & 3.17 & 1.78 & 1.87 \\
\hline $\mathrm{K}_{2} \mathrm{O}$ & 0.03 & 0.06 & 0.04 & 0.06 & 0.05 & 0.04 & 0.08 & 0.05 & 0.01 & 0.01 & 0.02 & 0.06 & 0.10 & 0.06 & 0.03 & 0.07 & 0.07 & 0.10 & 0.05 & 0.13 & 0.06 & 0.04 & 0.03 \\
\hline MgO & & & & & & & & 0.25 & 0.17 & & & & & & & & & & & & & & \\
\hline $\mathrm{FeO}$ & 0.50 & 0.37 & 0.44 & 0.83 & 0.51 & 0.49 & 0.36 & 0.54 & 0.42 & & & 1.02 & 0.89 & 0.62 & 0.39 & 0.52 & 0.57 & 1.04 & 0.70 & 0.83 & 0.62 & 0.32 & 0.41 \\
\hline Total & 99.68 & 100.47 & 100.67 & 99.02 & 98.69 & 99.74 & 100.02 & 99.11 & 98.21 & 99.31 & 98.66 & 100.03 & 100.58 & 99.59 & 101.13 & 100.32 & 101.05 & 100.39 & 101.06 & 100.49 & 100.33 & 99.57 & 99.40 \\
\hline \multicolumn{24}{|c|}{$\begin{array}{l}\mathrm{Nb} \text { cations } \\
\text { pet } 8 \text { oxygens }\end{array}$} \\
\hline $\mathrm{Si}$ & 2.224 & 2.293 & 2.247 & 2.396 & 2.244 & 2.237 & 2.225 & 2.325 & 2.223 & 2.282 & 2.152 & 2.422 & 2.411 & 2.305 & 2.148 & 2.216 & 2.226 & 2.438 & 2.344 & 2.487 & 2.234 & 2.185 & 2.200 \\
\hline Al & 1.735 & 1.670 & 1.722 & 1.580 & 1.740 & 1.732 & 1.752 & 1.650 & 1.758 & 1.706 & 1.826 & 1.538 & 1.552 & 1.671 & 1.831 & 1.760 & 1.745 & 1.523 & 1.633 & 1.472 & 1.635 & 1.796 & 1.770 \\
\hline $\mathrm{Ca}$ & 0.846 & 0.773 & 0.808 & 0.653 & 0.782 & 0.817 & 0.824 & 0.704 & 0.794 & 0.759 & 0.875 & 0.578 & 0.623 & 0.730 & 0.873 & 0.801 & 0.804 & 0.574 & 0.683 & 0.551 & 0.735 & 0.842 & 0.844 \\
\hline $\mathrm{Na}$ & 0.168 & 0.239 & 0.192 & 0.303 & 0.197 & 0.184 & 0.164 & 0.266 & 0.187 & 0.238 & 0.164 & 0.416 & 0.379 & 0.257 & 0.135 & 0.209 & 0.205 & 0.444 & 0.305 & 0.464 & 0.280 & 0.159 & 0.168 \\
\hline K & 0.002 & 0.003 & 0.002 & 0.004 & 0.003 & 0.002 & 0.005 & 0.003 & 0.001 & 0.001 & 0.001 & 0.003 & 0.006 & 0.004 & 0.002 & 0.004 & 0.004 & 0.006 & 0.003 & 0.007 & 0.003 & 0.002 & 0.002 \\
\hline $\mathrm{Mg}$ & & & & & & & & 0.017 & 0.012 & & & & & & & & & & & & & & \\
\hline $\mathrm{Fe}$ & 0.019 & 0.014 & 0.017 & 0.032 & 0.020 & 0.019 & 0.014 & 0.021 & 0.017 & & & 0.039 & 0.034 & 0.024 & 0.015 & 0.020 & 0.022 & 0.039 & 0.026 & 0.031 & 0.024 & 0.012 & 0.016 \\
\hline Total & 4.994 & 4.993 & 4.988 & 4.967 & 4.986 & 4.990 & 4.983 & 4.986 & 4.991 & 4.985 & 5.018 & 5.008 & 5.005 & 4.990 & 5.005 & 5.010 & 5.006 & 5.025 & 4.994 & 5.013 & 5.001 & 4.998 & 4.999 \\
\hline$\%$ An & 81.74 & 75.05 & 79.29 & 65.89 & 78.04 & 80.02 & 81.91 & 69.63 & 78.61 & 76.05 & 84.13 & 55.73 & 59.78 & 72.00 & 85.93 & 77.47 & 77.68 & 53.95 & 67.15 & 52.28 & 70.54 & 82.79 & 82,02 \\
\hline \multicolumn{24}{|c|}{ Sample 53417A-86-3 } \\
\hline & 70 & 71 & 72 & 73 & 74 & 75 & 76 & 77 & 78 & 79 & 80 & 81 & 82 & 83 & 84 & 85 & 86 & 87 & 88 & 89 & 90 & 91 & 92 \\
\hline $\mathrm{SiO}_{2}$ & 49.25 & 47.95 & 47.60 & 47.92 & 54.89 & 51.14 & 50.96 & 50.45 & 49.83 & 48.43 & 48.53 & 51.80 & 51.12 & 50.89 & 49.24 & 48.96 & 49.71 & 49.94 & 49.90 & 49.57 & 48.70 & 49.45 & 5026 \\
\hline $\mathrm{Al}_{2} \mathrm{O}_{3}$ & 31.38 & 33.24 & 33.62 & 32.54 & 28.42 & 31.23 & 31.15 & 31.53 & 32.28 & 33.25 & 32.76 & 29.12 & 29.66 & 30.43 & 31.78 & 31.93 & 31.81 & 31.49 & 31.50 & 31.69 & 32.24 & 32.45 & 31.77 \\
\hline $\mathrm{CaO}^{\circ} \mathrm{O}$ & 15.90 & 17.05 & 17,47 & 16.92 & 11.66 & 14.73 & 14.83 & 14.62 & 15.32 & 16.17 & 16.70 & 13.49 & 14.30 & 14.36 & 16.34 & 16.18 & 15.04 & 14.64 & 14.76 & 15.00 & 15.39 & 15.28 & 15.00 \\
\hline $\mathrm{Na}_{2} \mathrm{O}$ & 2.39 & 2.04 & 1.79 & 2.21 & 4.99 & 3.43 & 3.38 & 2.88 & 2.52 & 1.96 & 2.24 & 3.99 & 3.40 & 3.22 & 2.27 & 2.44 & 2.71 & 2.43 & 2.83 & 2.79 & 2.36 & 2.55 & 2.79 \\
\hline $\mathrm{K}_{2} \mathrm{O}$ & 0.03 & 0.06 & 0.04 & 0.05 & 0.12 & 0.05 & 0.07 & 0.05 & 0.03 & 0.04 & 0.06 & 0.06 & 0.03 & 0.06 & 0.04 & 0.07 & 0.04 & 0.08 & 0.02 & 0.03 & 0.05 & 0.03 & 0.03 \\
\hline MgO & & & & & & & & & & & & & & & & & 0.29 & 0.43 & 0.25 & 0.24 & 0.21 & 0.24 & 0.21 \\
\hline $\mathrm{FeO}$ & 0.34 & 0.62 & 0.44 & 0.43 & 0.97 & 0.59 & 0.71 & 0.37 & 0.47 & 0.32 & 0.50 & 0.57 & 0.37 & 0.39 & 0.42 & 0.48 & 0.54 & 0.59 & 0.44 & 0.46 & 0.43 & 0.47 & 0.43 \\
\hline Total & 99.29 & 100.94 & 100.96 & 100.07 & 101.05 & 101.17 & 100.57 & 99.90 & 100.45 & 100.04 & 100.79 & 99.03 & 98.88 & 99.35 & 100.09 & 100.06 & 100.14 & 99.60 & 99.70 & 99.78 & 99.38 & 100.47 & 100.29 \\
\hline \multicolumn{24}{|c|}{$\begin{array}{l}\text { Nb cations } \\
\text { per } 8 \text { oxygens }\end{array}$} \\
\hline $\mathrm{Si}$ & 2.269 & 2.172 & 2.170 & 2.203 & 2.464 & 2.310 & 2.30 & 2.301 & 2.26 & 2.212 & 2.21 & 2.383 & 2.355 & 2.334 & 2.254 & 2.244 & 2.269 & 2.286 & 2.284 & 2.270 & 2.241 & 2.249 & 2.278 \\
\hline $\mathrm{Al}$ & 1.704 & 1.801 & 1.806 & 1.763 & 1.503 & 1.662 & 1.661 & 1.695 & 1.729 & 1.790 & 1.760 & 1.579 & 1.711 & 1.645 & 1.715 & 1.725 & 1.711 & 1.699 & 1.699 & 1.711 & 1.748 & 1.739 & 1.704 \\
\hline $\mathrm{Ca}$ & 0.785 & 0.840 & 0.853 & 0.833 & 0.561 & 0.713 & 0.7 & 0.714 & 0.746 & 0.791 & 0.81 & 0.665 & 0.706 & 0.706 & 0.801 & 0.795 & 0.736 & 0.718 & 0.724 & 0.736 & 0.759 & 0.744 & 0.731 \\
\hline $\mathrm{Na}$ & 0.214 & 0.182 & 0.179 & 0.197 & 0.434 & 0.300 & 0.297 & 0.255 & 0.222 & 0.174 & 0.198 & 0.356 & 0.304 & 0.286 & 0.201 & 0.217 & 0.240 & 0.216 & 0.251 & 0.248 & 0.211 & 0.221 & 0.246 \\
\hline K & 0.002 & 0.004 & 0.004 & 0.003 & 0.007 & 0.003 & 0.004 & 0.003 & 0.002 & 0.002 & 0.003 & 0.004 & 0.002 & 0.004 & 0.002 & 0.004 & 0.003 & 0.005 & 0.001 & 0.002 & 0.003 & 0.001 & 0.001 \\
\hline $\mathrm{Mg}$ & & & & & & & & & & & & & & & & & 0.020 & 0.030 & 0.017 & 0.017 & 0.015 & 0.016 & 0.014 \\
\hline $\mathrm{Fe}$ & 0.013 & 0.024 & 0.017 & 0.017 & 0.036 & 0.022 & 0.027 & 0.014 & 0.018 & 0.012 & 0.019 & 0.022 & 0.014 & 0.015 & 0.016 & 0.018 & 0.021 & 0.023 & 0.017 & 0.018 & 0.016 & 0.016 & 0.018 \\
\hline Total & 4.986 & 5.021 & 5.007 & 5.016 & 5.005 & 5.011 & 5.004 & 4.981 & 4.982 & 4.981 & 5.008 & 5.008 & 4.992 & 4.989 & 4.990 & 5.004 & 4.998 & 4.975 & 4.993 & 5.000 & 4.993 & 4.998 & 4.990 \\
\hline$\%$ An & 77.49 & 80.15 & 82.73 & 79.33 & 54.05 & 68.69 & 68.67 & 72.41 & 75.51 & 80.80 & 78.76 & 63.51 & 68.81 & 69.83 & 78.53 & 76.89 & 72.25 & 72.52 & 71.70 & 72.19 & 75.62 & 74.04 & 72.46 \\
\hline
\end{tabular}


TABLE 2 - Continued

\begin{tabular}{|c|c|c|c|c|c|c|c|c|c|c|c|c|c|}
\hline & \multicolumn{11}{|c|}{ Sample 53-418A-86-3 } & \multicolumn{2}{|c|}{$\begin{array}{c}\text { Sample 45-395A- } \\
15.1\end{array}$} \\
\hline & 93 & 94 & 95 & 96 & 97 & 98 & 99 & 100 & 101 & 102 & 103 & 104 & 105 \\
\hline $\mathrm{SiO}_{2}$ & 50.41 & 48.50 & 48.28 & 48.00 & 49.08 & 48.77 & 49.71 & 51.72 & 48.98 & 47.90 & 49.87 & 52.36 & 47.21 \\
\hline $\mathrm{Al}_{2} \mathrm{O}_{3}$ & 32.10 & 32.97 & 32.68 & 32.68 & 32.07 & 32.37 & 31.65 & 30.89 & 31.50 & 32.24 & 31.24 & 30.11 & 34.02 \\
\hline & 14.90 & 16.15 & 15.96 & 16.10 & 15.27 & 16.21 & 15.47 & 14.32 & 15.29 & 16.18 & 15.13 & 13.05 & 16.99 \\
\hline $\mathrm{Na}_{2} \mathrm{O}$ & 2.85 & 2.08 & 2.41 & 2.03 & 2.41 & 2.10 & 2.46 & 2.92 & 240 & 2.03 & 2.64 & 3.82 & 1.65 \\
\hline $\mathrm{K}_{2} \mathrm{O}$ & 0.04 & 0.03 & 0.03 & 0.03 & 0.03 & 0.05 & 0.04 & 0.02 & 0.04 & 0.03 & 0.02 & 0.11 & 0.04 \\
\hline $\mathrm{MgO}$ & 0.25 & 0.20 & 0.22 & 0.18 & 0.27 & 0.19 & 0.21 & 0.34 & 0.21 & 0.19 & & 0.23 & 0.28 \\
\hline $\mathrm{FeO}$ & 0.43 & 0.41 & 0.46 & 0.43 & 0.48 & 0.41 & 0.43 & 0.56 & 0.41 & 0.51 & & 0.59 & 0.29 \\
\hline Total & 100.98 & 100.34 & 100.04 & 99.45 & 99.61 & 100.10 & 99.97 & 100,70 & 98.83 & 99.28 & 98.90 & 100.27 & 100.48 \\
\hline \multicolumn{14}{|c|}{$\begin{array}{l}\text { Nb cations } \\
\text { per } 8 \text { oxygens }\end{array}$} \\
\hline Si & 2.278 & 2.214 & 2.214 & 2.211 & 2.251 & 2.232 & 2.272 & 2.337 & 2.265 & 2.213 & 2.296 & 2.373 & 2.158 \\
\hline Al & 1.700 & 1.774 & 1.766 & 1.774 & 1.734 & 1.746 & 1.705 & 1.641 & 1.716 & 1.766 & 1.695 & 1.608 & 1.808 \\
\hline c: & 0.722 & 0.790 & 0.7 & 0.7 & 0.750 & & 0.757 & 0.693 & 0.757 & 0.801 & 0.746 & 0.634 & 0.832 \\
\hline $\mathrm{N}$ & 0.250 & 0.184 & 0.214 & 0.181 & 0.214 & 0.187 & 0.218 & 0.256 & 0.215 & 0.182 & 0.236 & 0.336 & 0.146 \\
\hline K & 0.003 & 0.002 & 0.002 & 0.002 & 0.002 & 0.003 & 0.003 & 0.001 & 0.003 & 0.002 & 0.001 & 0.007 & 0.003 \\
\hline $\mathrm{Mg}_{\mathrm{g}}$ & 0.017 & 0.014 & 0.015 & 0.013 & 0.018 & 0.013 & 0.017 & 0.023 & 0.015 & 0.013 & & 0.016 & 0.019 \\
\hline $\mathrm{Fe}$ & 0.016 & 0.016 & 0.018 & 0.016 & 0.018 & 0.016 & 0.015 & 0.021 & 0.016 & 0.020 & & 0.023 & 0.011 \\
\hline Total & 4.995 & 4.992 & 5.012 & 4.992 & 4.991 & 4.990 & 4.986 & 4.972 & 4.987 & 4.996 & 4.975 & 4.994 & 4.977 \\
\hline$\% \mathrm{An}$ & 71.68 & 78.65 & 75.93 & 78.91 & 74.75 & 78.38 & 74.95 & 69.72 & 75.25 & 78.68 & 75.89 & 62.40 & 82.30 \\
\hline
\end{tabular}




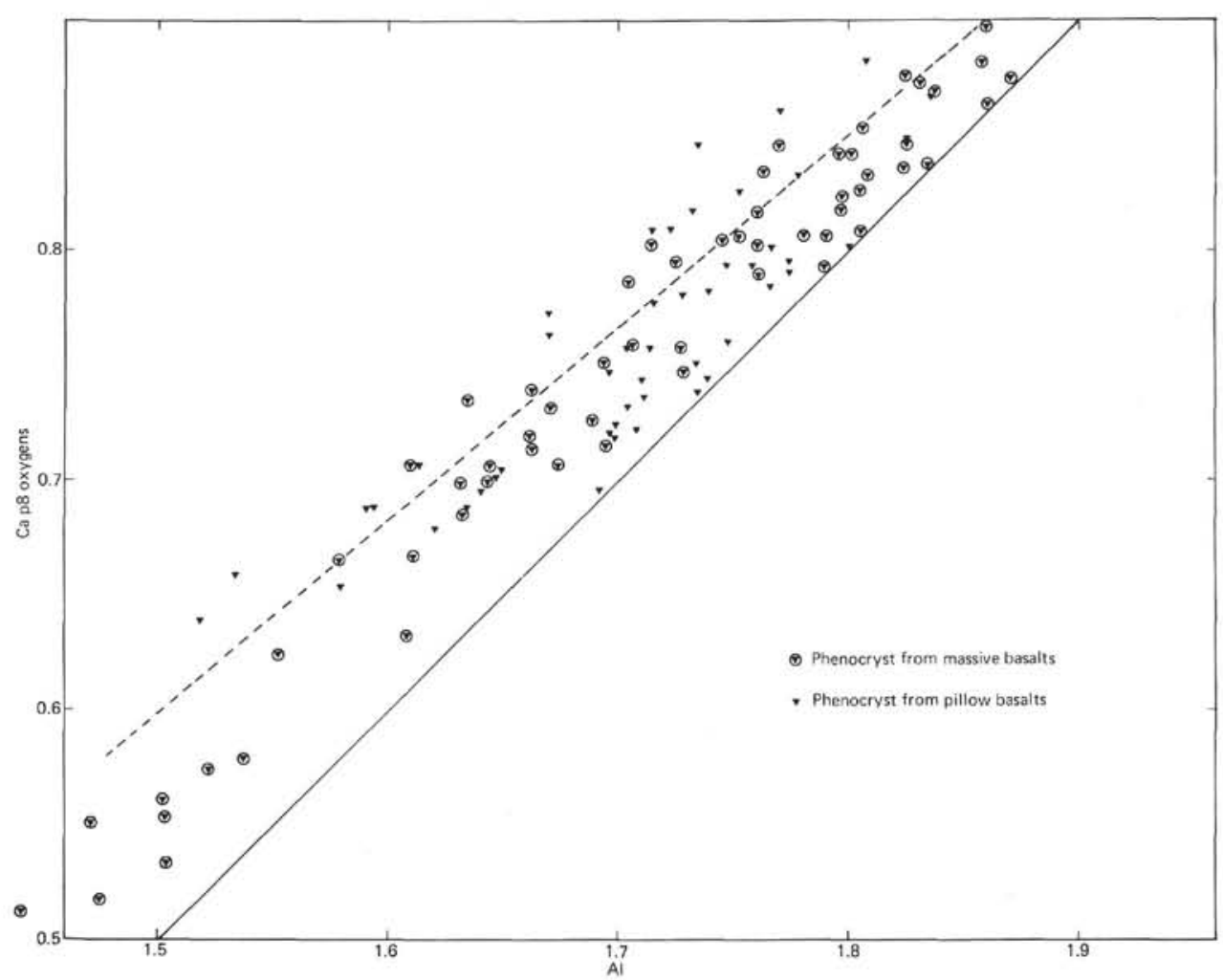

Figure 1. Number of Ca versus number of A1 atoms for 8 oxygens in the formula unit. The theoretical Ab-An solid solution is shown as a heavy line. The average line of Bryan's (1974) analyses is shown as a dashed line.

Bottinga, Y., Kudo, A.M., and Weill, D., 1966. Some observations on oscillatory zoning and crystallization of magmatic plagioclase, Am. Mineralogist, v. 51, p. $792-806$.

Bryan, W.B., 1974. Fe-Mg relationships in sector zoned submarine basalt plagioclase, Earth Planet. Sci. Lett., v. 24, p. 157-165.

Clocchiatti, R., Havette, A., Weiss, J., and Wilhelm, S., 1978. Les Bytownites du Rift d'Asal. Première partie. Etude des verres basaltiques inclus dans les mégacristaux: nouvelle approche pour la connaissance de certains processus pétrogénétiques, Bull. Soc. Franc. Mineral. Cryst., v. 101, p. 66-76.

Kirkpatrick, R.J., 1976. Towards a kinetic model for the crystallization of magma bodies, J. Geophys. Res., v. 81, p. 25652571.
1977. Nucleation and growth of plagioclase, Makaopuhi and Alae lava lakes, Kilauea Volcano Hawaii, Geol. Soc. Am. Bull., v. 87, p. $78-84$.

Shimizu, N., 1978. Analysis of zoned plagioclase from different magmatic environment a preliminary ion microprobe study, Earth Planet. Sci. Lett., v. 39, p. 398-406.

Sibley, D.F., Vogel, J.A., Walker, B.M., and Byerly, G., 1976. The origin of oscillatory zoning in plagioclase: a diffusion growth controlled model, Am. J. Sci., v. 276, p. 275-284.

Smith, J.V., 1974. Feldspar minerals: Berlin (Springer Verlag), v. 2 (1974).

Wright, T.L. and Kinoshita, W.T., 1968. March 1965 eruption of Kilauea Volcano and the formation of Makaopuhi Lava Lake, J. Geophys. Res., v. 73, p. 3181-3205. 

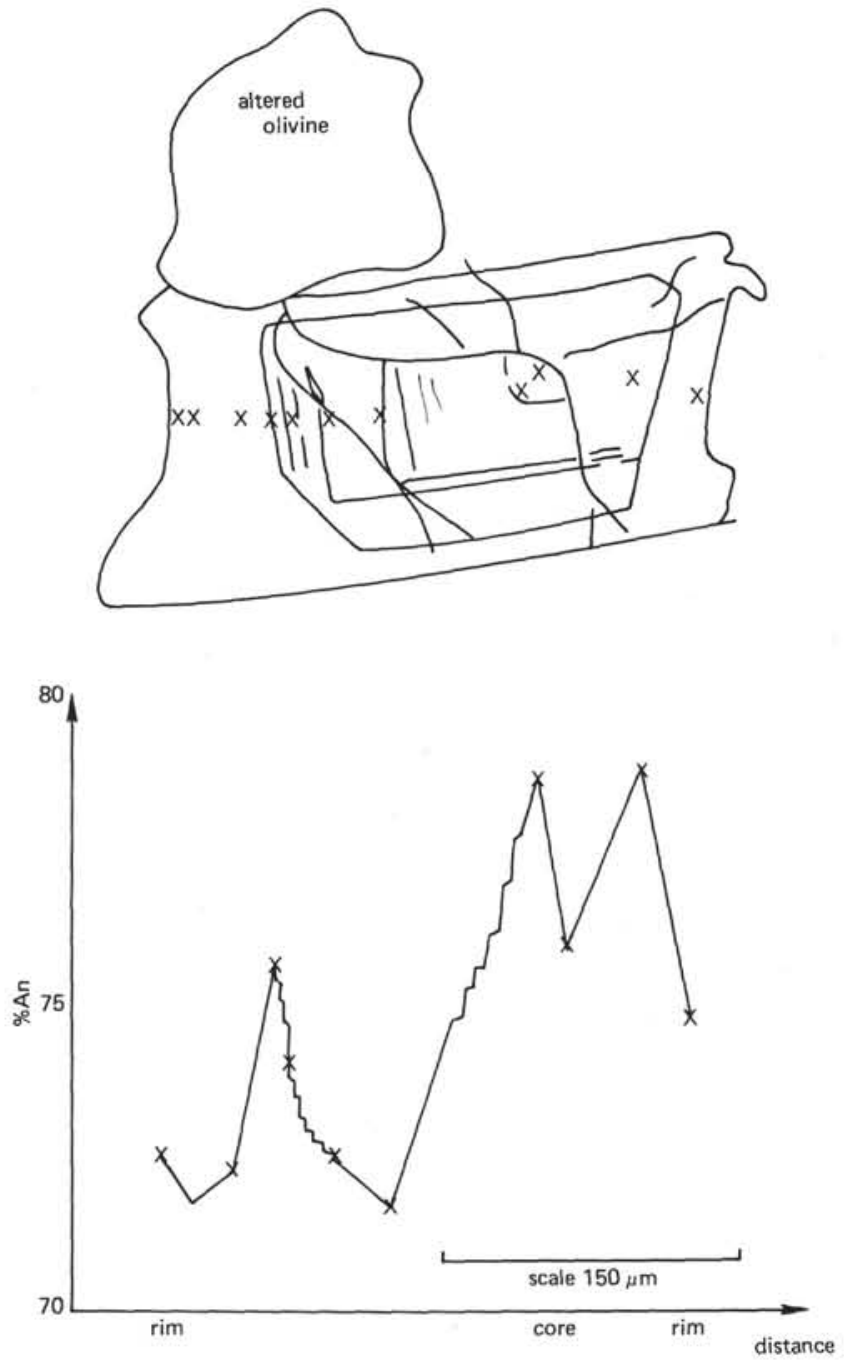

Figure 2. Sketch of the crystal represented by Analyses 86 to 97, Table 2, and variation of its chemical composition along the traverse shown by crosses. 